\title{
Theoretical analysis of flux amplification by soft magnetic material in a putative biological magnetic-field receptor
}

\author{
Valera P. Shcherbakov \\ Geophysical Observatory “Borok,” Russian Academy of Sciences
}

Michael Winklhofer*

Department of Earth and Environmental Sciences, Geophysics Section, Ludwig-Maximilians-University of Munich, Theresienstrasse 41, D80333, Munich, Germany

(Received 30 October 2009; revised manuscript received 25 January 2010; published 26 March 2010)

\begin{abstract}
Birds are endowed with a magnetic sense that allows them to detect Earth's magnetic field and to use it for orientation. Physiological and behavioral experiments have shown the upper beak to host a magnetoreceptor. Putative magnetoreceptive structures in the beak are nerve terminals that each contain a dozen or so of micrometer-sized clusters of superparamagnetic nanocrystals made of magnetite/maghemite and numerous electron-opaque platelets filled with a so far unidentified, amorphous ferric iron compound. The platelets typically form chainlike structures, which have been proposed to function as magnetic flux focusers for detecting the intensity of the geomagnetic field. Here, we test that proposition from first principles and develop an unconstrained model to determine the equilibrium distribution of magnetization along a linear chain of platelets which we assume to behave magnetically soft and to have no magnetic remanence. Our analysis, which is valid for arbitrary values of the intrinsic magnetic susceptibility $\chi$, shows that $\chi$ needs to be much greater than unity to amplify the external field by two orders of magnitude in a chain of platelets. However, the high amplification is confined to the central region of the chain and subsides quadratically toward the ends of the chain. For large values of $\chi$, the possibility opens up of realizing magnetoreceptor mechanisms on the basis of attraction forces between adjacent platelets in a linear chain. The force in the central region of the chain may amount to several $\mathrm{pN}$, which would be sufficient to convert magnetic input energy into mechanical output energy. The striking feature of an ensemble of platelets is its ability to organize into tightly spaced chains under the action of an external field of given strength. We discuss how this property can be exploited for a magnetoreception mechanism.
\end{abstract}

DOI: 10.1103/PhysRevE.81.031921

PACS number(s): 87.50.cf, 91.25.fa, 75.70.Kw

\section{INTRODUCTION}

A large number of animals, notably birds, have been reported to be capable of extracting information from the comparably weak geomagnetic field and using it for orientation, see [1-3] for reviews. Although the biological structures underlying the magnetic sense have not been identified with certainty yet, candidate magnetoreceptors on the basis of biogenic magnetite have been described in the nose of trout $[4,5]$ and in the upper-beak skin of homing pigeons $[6,7]$. Evidence for the involvement of the upper beak in magneticfield detection comes from behavioral experiments [8-10] and electrophysiological recordings from the trigeminal nerve [11], see also [12] for a recent review. Interestingly, the upper beak is not the only site of magnetic field reception, as birds are still able to obtain directional information from the magnetic field when the trigeminal nerve was sectioned to interrupt the information pathway from the beak to the brain [13].

The magnetite-based candidate magnetoreceptor structures in the pigeon beak are found in terminals of the trigeminal nerve [7]. These distinct terminals each contain about 20 clusters of densely packed superparamagnetic (SP)

\footnotetext{
*michaelw@LMU.DE

http://www.geophysik.uni-muenchen.de/michael
}

nanocrystals of magnetite/maghemite with grain sizes not larger than $5 \mathrm{~nm}[6,14]$. Within a terminal, the SP clusters are arranged along the long axis of the terminal $[7,15]$, which gives rise to anisotropic magnetic susceptibility. In other words, a group of SP clusters has a tendency to align parallel to the external field axis rather than perpendicular to it. According to theoretical models [15-18], this tendency may be exploited to produce a mechanical response to a change in magnetic field direction so that magnetic input energy may be converted into mechanical output energy. By way of mechanosensory transducers, such as strain-sensitive ion channels, the magnetic field change may eventually be transduced into a nerve signal (e.g., [15]).

Recently, the focus has shifted to conspicuous structural features that have been co-localized with the SP clusters in the putative avian magnetoreceptor in the beak; namely, numerous electron-opaque, iron bearing micron-sized chips of rectangular shape-referred to as "platelets"—arranged tightly one after another in the form of bands. The electron opaque platelets were found to be rich in iron and phosphorous, but amorphous [7]. Unlike the electron diffraction pattern for the SP nanocrystals, which had rings clearly attributable to magnetite or maghemite [14], the poor diffraction pattern for the platelets resembled that of amorphous or cryptocrystalline substances, which lack long-range order. Stahl and co-workers $[19,20]$ proposed that the electron-opaque chips represent maghemite monocrystals with a platy habitus 
a)

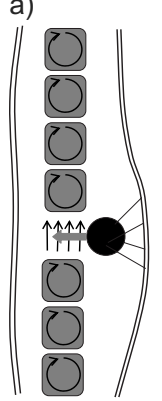

b)

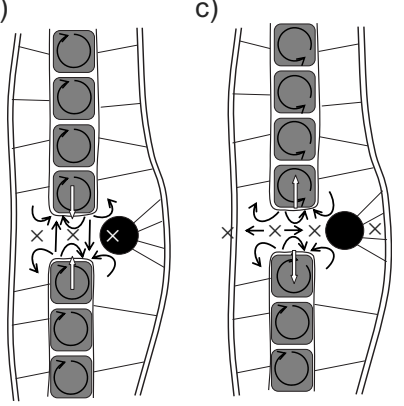

FIG. 1. Flux-amplifier model, (a) as proposed in [22] (see also [23]) with a highly simplified homogeneous stray-field distribution in the gap between two platelets in a chain of platelets. The proposed signaling element is a cluster of densely packed superparamagnetic nanocrystals (black sphere) that is anchored by elastic filaments to the unmyelinated membrane of the nerve terminal. The platelets (gray squares) are supposed to behave magnetically soft and to host magnetic vortex states (indicated by the circular arrows). Stray field (black arrows) produced by the platelet magnetization leaks into the gap and reinforces the flux density. The cluster nearby is attracted toward regions of higher stray field intensity (i.e., into the gap) and this force (white arrow) may be transmitted by the filaments to mechanosensitive ion channels in the nerve membrane. Sketches b) and c) show the stray field distribution that is qualitatively consistent with vortex magnetization configurations, for equal vortex-state chirality (b) and opposite chirality (c). The corners of a particle with vortex state are the major sources and sinks of stray field (black arrows). The actual stray field in the gap is quadrupolar, which implies annihilation points where the stray field is zero (crosses), here shown for zero external field. With increasing field strength parallel to the chain axis, the net magnetization of the platelets increases too (see also Fig. 3), which reinforces the stray field around the gap and makes it more asymmetric. This way, the force experienced by the cluster could be controlled by the external field strength. Note that in order for the mechanism to work as conceived, the platelets need to be anchored (indicated by filaments in $\mathrm{b}$ and $\mathrm{c}$ ) to prevent the chain segments from moving toward the cluster (because of reaction forces) and from moving toward or away from each other due to attractive (b) or repulsive (c) forces (white arrows).

of dimensions $1 \times 1 \times 0.1 \mu^{3}$. This proposition has been criticized because a micron-sized maghemite monocrystal would produce bright diffraction spots [21] but not the poor diffraction pattern reported earlier in [7].

There is no doubt that maghemite monocrystals - if present-would make a lot of sense in the context of magnetoreception. Fleissner et al. [22] proposed a conceptual model in which a chain of easily magnetizeable "maghemite platelets" functions as magnetic-flux concentrator, which locally amplifies the geomagnetic field and produces a strong stray field at either end of the chain or where the chain is interrupted (Fig. 1). An SP cluster that is close to such a stray-field source experiences an attraction force proportional to the gradient of $\left\langle H_{\mathrm{s}}(\mathbf{r})^{2}\right\rangle_{V}$, where $H_{\mathrm{s}}(\mathbf{r})$ is the stray field and $\langle.\rangle_{V}$ denotes the average over the volume $V$ of the SP cluster $\left(\begin{array}{lll}V \sim \mathrm{m}^{3}\end{array}\right)$. The magnetic gradient force can be transduced into a receptor potential with the aid of elastic filaments connecting the SP cluster to the mechanically sensitive membrane of the dendrite. It has not been shown whether the

platelet-cluster-interaction mechanism proposed in [22] achieves the sensitivity necessary to detect small variations in magnetic-field strength, which is considered the hallmark of the so-called magnetic map sense (see [3] for a review of magnetic maps in animals). Solov'yov and Greiner [24,25] tested the suitability of the mechanism for the avian compass sense. They assumed a "maghemite platelet" to magnetically behave like a single-domain (SD) particle in the sense that it is uniformly magnetized and has a magnetic moment whose magnitude is independent of the external field strength. The only degree of freedom granted to the magnetization of a platelet in that strongly constrained model is the orientation of the magnetization vector $\mathbf{M}$ within the plane, see Eq. (3) in [24] or Eq. (1) in [25]. That approach with a magnetic moment fixed in magnitude does therefore not exactly capture the idea conveyed in [22], who assumed easily magnetizeable platelets, where the net magnetic moment per platelet depends on the external field strength. Micromagnetic results suggest that a maghemite monocrystal of dimensions $1 \mu \mathrm{m}$ by $1 \mu \mathrm{m}$ by $0.1 \mu \mathrm{m}$, accommodates a four-domain (4D) (more precisely, a vortex) configuration rather than an SD state [26]. A 4D state has no magnetic remanence, but can be magnetized easily, a behavior that is commonly referred to as magnetically soft. Solov'yov and Greiner [27] used a micromagnetic model to compute the equilibrium magnetization in a chain of maghemite platelets in function of the number of platelets at 0.5 Oe (earth strength field, 40 $\mathrm{A} / \mathrm{m}$ in SI units). They found that chains consisting of more than six extremely tightly spaced platelets can sustain a magnetization configuration, in which the inner platelets are practically homogeneously magnetized, while the outer platelets host a 4D (vortex) state.

Here, we focus on the dependence of the magnetization of a chain of platelets on the external field intensity in order to assess the proposition by [22] that a chain of platelets could function as flux amplifier in the measurement of the intensity of an external field. However, since the exact composition of the platelets and their magnetic properties remain to be experimentally determined, we will not restrict ourselves to a certain type of material. The absence of a crystalline structure of the platelets [7] implies that the material in question has negligible magnetocrystalline anisotropy, hence we assume that the material in question is magnetically soft and can be described by its intrinsic magnetic susceptibility, $\chi$. For such a chain of soft platelets, we will derive analytical expressions for the unconstrained equilibrium magnetization of a chain on the basis of energy minimization principles. This way, our analysis will be valid for arbitrary values of $\chi$, which allows us to address a large range of candidate compositions.

\section{BASIC CONSIDERATIONS}

Before deriving analytical expressions for the equilibrium magnetization of a chain of magnetically soft platelets, we introduce the basic concepts by way of simple examples.

\section{A. Individual platelet}

It is instructive to first consider the magnetic behavior of an isolated magnetic platelet. The shape of a platelet is ap- 


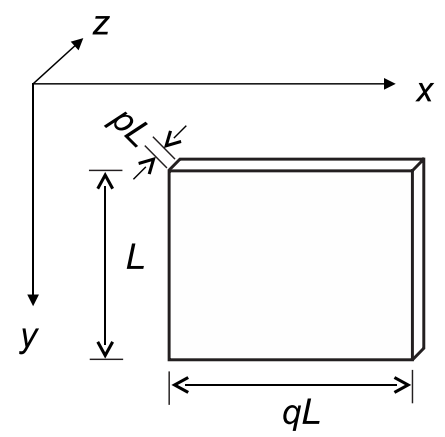

FIG. 2. Geometry of a platelet and coordinate system. A platelet is modeled as a rectangular body with dimensions $q L, L, p L$ along the $x, y, z$ axes, respectively.

proximated by that of a rectangular prism of length $q L$, width $L$, and thickness $p L$ (Fig. 2). According to [20], the typical dimensions are $L=1 \mu \mathrm{m}, q=1$, and $p=0.1$. We derive the magnetic energy under the assumption that it reversibly acquires an induced homogeneous magnetization $\mathbf{M}$ in a homogeneous external field $\mathbf{H}$, but does not retain a magnetic remanence. In other words, the platelets are considered to be magnetically very soft, as is the case for amorphous materials lacking long-range order. We can therefore neglect magnetocrystalline anisotropy energy and represent the total magnetic energy of such a platelet as the sum of externalfield (Zeeman) energy and demagnetizing energy,

$$
E(M)=L^{3} p q\left(-H M+\frac{1+N \chi}{2 \chi} M^{2}\right),
$$

(in c.g.s. units), where we assume without loss of generality that $\mathbf{H}$ is parallel to any of the sides of the platelet. Here, $\chi$ is the intrinsic magnetic susceptibility of the magnetic material within the platelet and $N$ is the demagnetization factor of the platelet. For example, if the magnetization is parallel to the side $q L$, i.e., $\mathbf{M}=M \mathbf{e}_{x}, N_{x}$ can be written according to [28] as

$$
N_{x}(p, q)=\frac{4}{p q}[F(p, 0)-F(p, q)],
$$

where $F(p, q)$ is the mutual energy (in terms of $L^{3} M^{2}$ ) of the two rectangles with sides $L$ and $p L$ lying opposite each other in parallel planes a distance $q L$ apart and $F(p, 0)$ is the self energy (in terms of $L^{3} M^{2}$ ) of each rectangle. The demagnetization factor for a platelet magnetized parallel to the side $p L$, i.e., $\mathbf{M}=M \mathbf{e}_{z}$,

$$
N_{z}(p, q)=\frac{4}{p q}[F(q, 0)-F(q, p)] .
$$

Finally, the demagnetization factor $N_{y}$ can be obtained from the generally valid relation

$$
N_{x}+N_{y}+N_{z}=4 \pi
$$

(in c.g.s.), or using the expression from Eq. (2), as

$$
N_{y}(p, q)=N_{x}(p / q, 1 / q) .
$$

Note that, due to symmetry reasons, $N_{y}(p, q)=N_{y}(q, p)$.

For a quadratic platelet $(q=1), N_{x}(p, 1)$ decreases from $4 \pi / 3$ to zero as the thickness $p$ goes from 1 to 0 (see first row in Table I). At the same time, $N_{z}(p, q)$ increases from $4 \pi / 3$ to $4 \pi$ as $p$ goes from 1 to 0 . For a thin platelet with $q=1$ and $p=0.1, N_{x} / N_{z}=8.26$, which implies that it can be magnetized much more easily in the $x y$ plane than perpendicular to it. It is therefore reasonable to neglect the out-ofplane magnetization component $M_{z}$ and to consider only the in-plane components $H_{x}, H_{y}$ of the external magnetic field.

Minimization of Eq. (1) with respect to $M$ yields the wellknown expression

$$
M=\frac{\chi}{1+N \chi} H \equiv \chi^{\prime} H,
$$

where $\chi^{\prime}$ is referred to as apparent susceptibility, which is the slope of the magnetization curve $M(H)$. Equation (6) can be simply derived when bearing in mind that $\chi H_{\text {eff }}=\chi^{\prime} H$, where $H_{\text {eff }}=H-N M$ is the effective magnetic field, which compared to the external magnetic field $H$ is reduced by the demagnetizing field $N M$. For $\chi \gg 1$, the demagnetization effect dominates and $\chi^{\prime} \cong 1 / N$. In the limit of vanishing $\chi$, the demagnetization vanishes, but that case is certainly not worth exploring in the context of a putative magnetoreceptor.

TABLE I. Demagnetizing factor $N_{x}(p, q) / N(1,1)$ of a platelet magnetized parallel to the side $q L$ for various length-to-width ratios $q$ and height-to-width ratios $p . N(1,1)=4 \pi / 3$ is the demagnetizing factor for an isometric body. See Eq. (2) for a definition of $N_{x}(p, q)$. Note that $\lim _{p \rightarrow 0} N_{x}(p, q)=0$. For $q \leq p, N_{x}$ is given in Table II. All values calculated with formulas derived in [28]. See also [29], pp. 122, or [30].

\begin{tabular}{rllllll}
\hline \hline$q / p$ & 1.0 & 0.8 & 0.6 & 0.4 & 0.2 & 0.1 \\
\hline 1 & 1 & 0.92 & 0.82 & 0.68 & 0.46 & 0.29 \\
2 & 0.60 & 0.54 & 0.47 & 0.38 & 0.25 & 0.16 \\
3 & 0.42 & 0.38 & 0.33 & 0.26 & 0.17 & 0.11 \\
4 & 0.33 & 0.30 & 0.25 & 0.20 & 0.13 & 0.081 \\
5 & 0.27 & 0.24 & 0.21 & 0.16 & 0.11 & 0.065 \\
6 & 0.22 & 0.20 & 0.17 & 0.14 & 0.089 & 0.055 \\
7 & 0.19 & 0.17 & 0.15 & 0.12 & 0.076 & 0.047 \\
13 & 0.11 & 0.095 & 0.082 & 0.065 & 0.042 & 0.026 \\
21 & 0.067 & 0.060 & 0.051 & 0.040 & 0.026 & 0.016 \\
\hline \hline
\end{tabular}




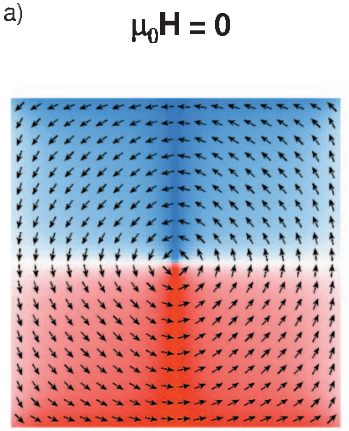

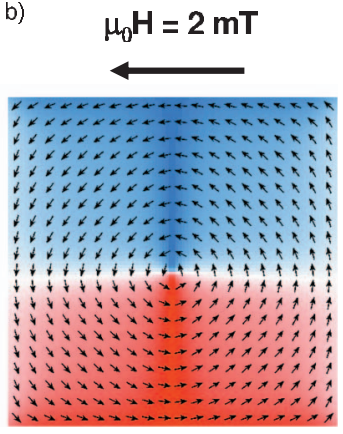

FIG. 3. (Color) Magnetic vortex structure in the $x, y$ plane of a platelet (dimensions of $L=1 \mu \mathrm{m}, p=0.1$, and $q=1$ ). The magnetization structure (arrows) has a counterclockwise sense of rotation about the core of the vortex. a) zero-field state: vortex core is in the center of the platelet, the blue and red domains have equal area and thus cancel each other so that the vortex has no net magnetization in the $(x, y)$ plane; b) at $H=20$ Oe $(1.6 \mathrm{kA} / \mathrm{m})$, the blue domain, which has magnetization components parallel to $\mathbf{H}$, is larger than the red domain, which has magnetization components antiparallel to H. The imbalance between red and blue domain produces a net magnetization parallel to $\mathbf{H}$.

We have assumed thus far that a platelet is uniformly magnetized. However, the dimensions of the platelets in the homing pigeons are conducive to the formation of nonuniform magnetization structures in weak magnetic fields, provided that the platelets are made of strongly magnetic material, such as magnetite or maghemite. Micromagnetic simulations [26] suggest that magnetic vortex structures are the most favorable magnetization states in thin micron-sized platelets of maghemite composition.

In the following, we will show that the formulas above can still be used for nonuniformly magnetized platelets that carry a magnetic vortex at earth-strength fields. We performed numerical micromagnetic modeling using the objectoriented micromagnetic framework (OOMMF) code [31] in order to determine the initial (apparent) magnetic susceptibility $\chi^{\prime}$ of isolated platelets and interacting platelets. Without loss of generality, we use material parameters for maghemite with zero magnetocrystalline anisotropy energy. As shown in [26], maghemite platelets are soft enough to host a vortex state, so from that point of view maghemite serves us as a convenient model to demonstrate the applicability of our general approach.

We found $\chi^{\prime}$ to be constant over the studied field range of -20 Oe to 20 Oe $( \pm 1.6 \mathrm{kA} / \mathrm{m})$. At zero field, the core of the vortex is centered in the platelets, leading to zero net magnetization [Fig. 3(a)]. As the field increases, the core of the vortex moves away from the center of the platelet, thereby making way for the domain that has a magnetization parallel to the field. The vortex moves as a stiff magnetization mode through the platelet, that is, the magnetization structure shifts as a whole without changing the relative orientation of adjacent spins [Fig. 3(b)]. Thereby, the exchange energy remains constant. From a Taylor expansion of the demagnetizing energy density about $M=0$,

$$
e_{d}(M) \cong e_{d}(0)+\frac{1}{2} \frac{\partial^{2} e_{d}}{\partial M^{2}} M^{2}+\ldots,
$$

we determine $\chi^{\prime}=\left(\partial^{2} e_{d} / \partial M^{2}\right)^{-1}$ as 1.0 c.g.s. $(4 \pi \mathrm{SI})$ for a platelet with $q=1$ and $p=0.1$. The value of $\chi^{\prime}$ determined this way exactly agrees with the slope of the initial magnetization curve. This identity confirms that the change in the total magnetic energy $E$ during the process of initial magnetization is entirely due to the changes in demagnetizing energy. Similar conclusions were reported by [32] who calculated magnetization curves for cylindrical nanodots. Remarkably, the value $\chi^{\prime}=1.0$ c.g.s. is even higher than that predicted by Eq. (6) for a uniform magnetization structure with $N_{x}(0.1,1)=1.22$ and $\chi^{\prime} \approx 1 / N_{x}(0.1,1)=0.816$. Thus, the vortex structure is actually more easily magnetized compared to a uniform distribution of $M$. Evidently, the difference is explained by the adaptation of the actual magnetic pole distribution (at a given net magnetization value $M$ ) to the vortex structure. Because the exchange energy does obviously play no role in the initial magnetization process-the vortex propagates as a stiff mode- the only energies involved are demagnetizing energy and Zeeman energy. In this context it is worth noting that the demagnetizing factor for a twodomain particle hosting a thin planar domain wall is only $40 \%$ of that for a uniformly magnetized particle [33-35].

From Eq. (1), we can now estimate the surplus magnetic energy stored in a platelet magnetized by an earth-strength field $(H=0.5 \mathrm{Oe}$, or $40 \mathrm{~A} / \mathrm{m})$ as

$$
E \approx-\frac{H^{2} L^{3} p q}{2 N_{x}(p, q)}
$$

which yields approximately $10^{-21} \mathrm{~J}$, or $0.25 k_{B} T$ where $k_{B} T$ is the thermal energy at room temperature. It is clear that a sensitive magnetoreception mechanism cannot be realized on the basis of an individual platelet hosting a magnetic vortex structure.

\section{B. Chains of platelets}

If we consider a pair of platelets, arranged one-afteranother along the $x$ axis, with a small gap between them, the gain in the total energy is more than twice compared to two isolated platelets. This gain is due to the magnetostatic interaction between the surfaces at either end of the gap. The demagnetization factor of such a pair can be roughly approximated by the one for a platelet of double size, because $N_{x}(0.1,2)=0.65=0.54 N_{x}(0.1,1)$. The total energy increases almost four times and now reaches $k_{B} T$. The situation becomes even more promising when a larger number $n$ of quadratic platelets is combined into a tightly spaced chain because the energy of the chain increases much faster than simply in direct proportion to the number $n$ of platelets in a chain due to both the increase in the total volume of platelets and decrease of the effective demagnetizing factor with increasing chain length.

For a tightly spaced chain of $n$ platelets, we now approximate the demagnetizing factor $N_{x}$ by the demagnetizing factor of a prism of length $n L$ and rectangular cross section $p L^{2}$, i.e., $N_{x}(p, n)$. For the case $p=0.1$, we find $N_{x}(0.1, n)$ 
$\approx 7 N_{x}(0.1,1) /(6 n+1)$, where the approximation deviates from the true value by not more than $1 \%$ for $n \geq 1$. Because the total volume now is $n p L^{3}$, the energy Eq. (8) becomes

$$
E_{n} \approx-\frac{n^{2}}{2} \frac{H^{2} p L^{3}}{N_{x}(0.1,1)} .
$$

Thus, the energy of interacting platelets is proportional to the square of the number $n$ of platelets in a chain and the square of the magnetic field. This characteristics is entirely different from that of a chain of magnetic SD particles, such as magnetosome chains in magnetic bacteria. In SD particles, the magnetization intensity is constant, which means that small variations of the external field strength against the background field will practically not change the interaction energy of the SD particles, which is proportional to the number of particles.

Thus, a chain of tightly spaced (strongly interacting), soft magnetic platelets appears to be a promising system for the purpose of amplification of the external magnetic field. Yet, we need to point out that this consideration is a best-case scenario, as we have neglected the effects of finite gaps between the platelets. Magnetic flux leaks through the gaps, and therefore, they will tend to diminish the magnetic field amplification.

\section{Converting the magnetic input energy into mechanic output energy}

So far we have considered the energetics of a chain of magnetically soft platelets in the external field, but in order for the chain to work as a magnetoreceptor, its magnetic energy has to be converted into a physiologically exploitable output signal.

\section{Magnetic torque: Compass needle}

The classical way of converting magnetic energy into mechanic energy takes advantage of the magnetic torque an elongated magnetic body — such as a compass needleexperiences in a magnetic field applied at an oblique angle. Let the external field $\left(H_{x}, H_{y}, 0\right)=H(\cos \phi, \sin \phi, 0)$, where $\phi$ is the angle between the magnetic field and chain axis. The chain has an induced magnetic moment $\mu_{\mathrm{tot}}=\left(M_{x}, M_{y}, 0\right) n V$, where $V=p L^{3}$ is the volume of an individual platelet. The magnetic torque $\mathbf{T}=\mu_{\text {tot }} \times \mathbf{H}$, is given by $T_{z}=n\left(M_{x} H_{y}\right.$ $\left.-M_{y} H_{x}\right) V$. Under the assumption that we can neglect the effect of finite gaps between the platelets, we can use the expression Eq. (6) for $M_{\nu}$ with $\nu=x, y$ and obtain

$$
T_{z}=\frac{1}{2}\left(\chi_{x}^{\prime}-\chi_{y}^{\prime}\right) n V H^{2} \sin 2 \phi .
$$

For large values of $\chi, \chi_{\nu}^{\prime} \approx 1 / N_{\nu}(p, n)$, which yields

$$
T_{z}(n) \approx \frac{1}{2} \frac{n(n-1)}{N_{x}(0.1,1)} H^{2} V \sin 2 \phi,
$$

for $n$ identical platelets with $p=0.1$ and $q=1$. Thus, the torque depends quadratically on the number of platelets, in other words, a torque-based mechanism can take advantage

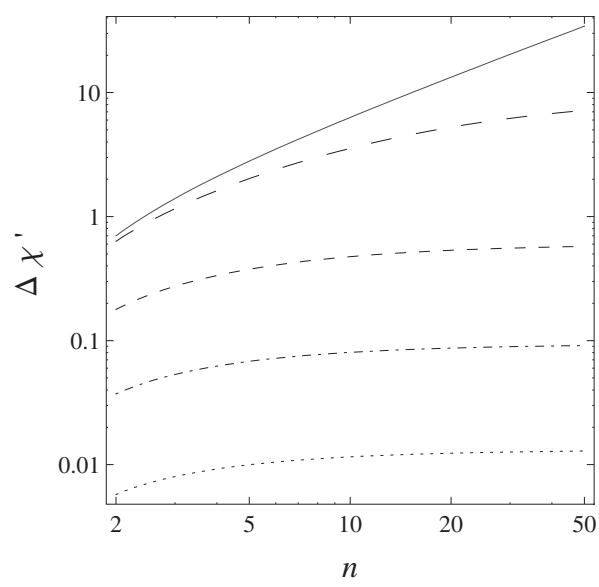

FIG. 4. Magnetic torque pre-factor $\Delta \chi^{\prime}=\left(\chi_{x}^{\prime}-\chi_{y}^{\prime}\right)$ [Eq. (10)] as a function of the number $n$ of platelets in a chain, for various values of $\chi$. From top to bottom: $0.7(n-1)$ (gray solid) as in Eq. (11) for $1 / \chi=0 ; \chi=10$ (long dashes); $\chi=1$ (short dashes); $\chi=0.3$ (dot dashed); $\chi=0.1$ (dotted). Note the similarity among the $\Delta \chi^{\prime}$ curves for $\chi \leq 1$, which converge rapidly toward the limit value of $\Delta \chi^{\prime}$ $\approx \chi^{2}$ for $n \gg 1$.

of the nonlinear amplification of the external field, provided that the platelets have a large intrinsic susceptibility and are tightly spaced. Similarly, the torque $T_{y}$ the chain experiences in a magnetic field tilted by an angle $I$ in the $(x, z)$ plane is obtained as

$$
T_{y}(n) \approx-E_{n} \sin 2 I .
$$

Like the energy, the maximum torque $\left|T_{y}\left(I=45^{\circ}\right)\right|$ exceeds the thermal energy by one order of magnitude once $n>6$.

It appears straightforward to realize a biological compass with a chain of magnetically very soft platelets, although we need to point out that the torque considerations are-as were the energy considerations further above-are best-case scenarios, neglecting the effect of a finite gap between adjacent platelets in a chain and assuming ideal soft-magnetic behavior $(1 / \chi \rightarrow 0)$. The effect of a finite gap between adjacent plaletes in a chain on the torque will be considered later.

We shortly consider the case that the magnetic material in the chain has a magnetic susceptibility comparable to a SP system, say $\chi \leq 1$ c.g.s., then $\left(\chi_{x}^{\prime}-\chi_{y}^{\prime}\right)$ in Eq. (10) is practically independent of $n$ for $n>10$ (Fig. 4) and $T_{x}$ scales with $n \chi^{2} H^{2}$. The same scaling relation was obtained for the torque acting on a linear chain of SP clusters $[16,17])$.

\section{Magnetic attraction force between adjacent platelets: Magnetometer}

Having shown a chain of magnetically soft platelets to be viable as compass needle, we next explore whether it also has the potential to act as a magnetometer. The torque depends (nonlinearly) on both the field angle ( $\phi$ or $I$ ) and intensity, which precludes a simultaneous measurement of both quantities independently. If, however, the chain happens to be oriented along or is actively rotated into a maximum torque position (at $\phi=45^{\circ}$ or $I=45^{\circ}$ ), it is in principle possible to isolate $H^{2}$. 
TABLE II. Demagnetizing factor $N_{x}(p, r)$ of a narrow gap $(r \leq p=0.1)$ between two rectangles (of unit width and fixed height-to-width ratio $p$ ) magnetized along the $x$ axis and separated by a gap of size $r$. Note that $\lim _{r \rightarrow 0} N_{x}(p, r)=4 \pi$. See Eq. (11) for a definition of $N_{x}(p, r)$.

\begin{tabular}{lccccccc}
\hline \hline$r$ & 0.001 & 0.005 & 0.02 & 0.04 & 0.06 & 0.08 & 0.1 \\
\hline$N_{x}$ & 12.3 & 11.6 & 9.92 & 8.47 & 7.43 & 6.63 & 6.00 \\
\hline \hline
\end{tabular}

In the following, we discuss another mechanism of measuring $H^{2}$, which works best for a chain that is coaxial with the external magnetic field (i.e., zero torque position at $\phi$ $=I=0^{\circ}$ ). The mechanism is based on the magnetic attraction force between adjacent platelets in a chain. First, we consider the magnetic energy contained in a gap of width $r L$ between two adjacent platelets that have the same magnetization $M$ and same surface area $p L^{2}$. We can now assign a demagnetizing factor to the gap,

$$
N_{r}:=N_{x}(p, r)=4[F(p, 0)-F(p, r)] /(p r)
$$

(see Table II for numerical values). The magnetostatic self energy of the gap then is given by

$$
W_{\mathrm{ms}}=\frac{1}{2} N_{r} M^{2} r p L^{3},
$$

from which we can derive the magnetic attraction force as

$$
F_{\mathrm{ms}}=\frac{d W_{\mathrm{ms}}}{L d r} \cong \frac{1}{2} N_{r} M^{2} p L^{2},
$$

where we have neglected the term containing $r d N_{r} / d r$ on the right-hand side, because $N_{r}(p, r)$ converges very slowly toward its limit value of $4 \pi$ when $r \rightarrow 0$ and because $r$ is small anyway. It is interesting to compare the expression for $F_{\mathrm{ms}}$ with the force between two adjacent single-domain particles in a magnetosome chain, $F_{\mathrm{ms}}^{M_{s}}=2 \pi a^{2} M_{s}^{2}$ [36], where $M_{s}$ is the saturation magnetization and $a$ the edge length of a magnetosome crystal. With $M_{s}=480 \mathrm{G}$ for magnetite (4.8 $\left.\times 10^{5} \mathrm{~A} / \mathrm{m}\right)$ and $a=40 \mathrm{~nm}, F_{\mathrm{ms}}^{M_{s}}=230 \mathrm{pN}$. However, since magnetosomes almost always carry their saturation magnetization $F_{\mathrm{ms}}^{M_{s}}$ is constant and therefore cannot be used to detect the strength of the external magnetic field (chains of magnetosomes are optimized for the torque mechanism). This is the fundamental difference to a chain of magnetically soft platelets, where $F_{\mathrm{ms}}$ depends on $H^{2}$. According to Eq. (13), the corresponding forces will be of the order of $2 \pi(M / H)^{2} 10^{-2} \mathrm{pN} / \mathrm{Oe}^{2}$. Relevant biological forces are of the order of $\mathrm{pN}$ and therefore we require that $M / H \geq 8$ to ensure that $F_{\mathrm{ms}} \geq 1 \mathrm{pN}$ (at $H \sim 0.5 \mathrm{Oe}$ ). The quantity $M / H$ $\equiv \alpha$ obviously defines a magnetic field amplification factor. To determine $\alpha$, we first need to derive the equilibrium magnetization distribution along the chain axis. This will be performed in the following section. Readers who are less interested in the technical details can jump directly to the section "Results."

\section{MATHEMATICAL ANALYSIS}

We now consider a one-dimensional chain of $n$ identical quadratic platelets, each of size $(L, L, p L)$, with a narrow gap $r_{i} L$ between the platelets along the chain-axis ( $x$ axis). Since $r_{i} \ll p \ll 1$, the only significant contributions to the demagnetizing energy come from self energy of the surfaces having nonzero pole density and the interactions between the pairs of surfaces either side of the gaps. Extending Eq. (1) to $n$ particles, the total energy can be written as

$$
\begin{aligned}
E= & p L^{3}\left[-H \sum_{i=1}^{n} M_{i}+\frac{N_{x}(p, 1)}{2} \sum_{i=1}^{n} M_{i}^{2}-2 \sum_{i=1}^{n-1} M_{i} M_{i+1} \frac{F\left(p, r_{i}\right)}{p}\right. \\
& \left.+\frac{1}{2 \chi} \sum_{i=1}^{n} M_{i}^{2}\right],
\end{aligned}
$$

where we consider only nearest-neighbor interactions and neglect interactions with next-nearest and higher-order neighbors, which fall off quickly with distance (i.e., as ( $i$ $-j)^{-3}$ ) and therefore contribute only little to the total energy.

Since $F(p, 1)=0.0047 \ll F(p, 0)=0.035$ for $p=0.1$, we can approximate $N_{x}(p, 1)$ by $N_{p}$,

$$
N_{p}=4 F(p, 0) / p \approx N_{x}(p, 1) .
$$

That approximation is reasonable, since $N_{p} \approx \sqrt{2}$ for $p=0.1$ and $N_{x}(0.1,1) \approx 1.23$.

Introducing dimensionless energy, $E / E_{0}$ with $E_{0}=L^{3} p H^{2}$, and dimensionless magnetizations $m_{i}=M_{i} / H$, Eq. (14) becomes

$$
\begin{aligned}
e= & -\sum_{i=1}^{n} m_{i}+\frac{1}{2} N_{p} \times\left[\left(1+\frac{1}{N_{p} \chi}\right) \sum_{i=1}^{n} m_{i}^{2}\right. \\
& \left.-\sum_{i=1}^{n-1} f\left(p, r_{i}\right) m_{i} m_{i+1}\right],
\end{aligned}
$$

with

$$
f\left(p, r_{i}\right)=\frac{F\left(p, r_{i}\right)}{F(p, 0)} \leq 1 .
$$

Generally speaking, the magnetic properties of a chain of small objects like the micron-sized platelets must be computed by means of statistical physics. As shown in Appendix A, the thermodynamic and mechanical equilibrium values of $M_{i}$ are exactly the same, hence, they can be calculated simply by minimization of the energy function Eq. (16). The maximum magnetization values can be obtained at $1 / \chi \rightarrow 0$ and $r_{i}=0$, i.e., when $f\left(p, r_{i}\right)=1$.

\section{Approximative analytical solutions}

From the physical point of view, it is more satisfying to derive an analytical solution for the magnetization distribu- 
tion in a chain of platelets. The analytical solutions presented in Appendix B are too nonlinear to convey a simple picture of the general magnetization behavior of a chain of platelets. It is therefore instructive to consider the two extreme cases of $\lambda n \ll 1$ and $\lambda n \gg 1$, where $\lambda$ is the characteristic nondimensional length scale of the problem,

$$
\lambda=\sqrt{\frac{2 N_{r}^{\prime}}{N_{p}}} .
$$

Here, $N_{r}^{\prime}$ is the effective demagnetization factor of a gap,

$$
N_{r}^{\prime}=\frac{1+\chi r N_{r}}{\chi} \text {. }
$$

\section{$\lambda n \ll 1$}

Since $n>1$, the condition $\lambda n \ll 1$ holds if $N_{r}^{\prime} \rightarrow 0$. Since $N_{r}^{\prime} \equiv 1 / \chi+4 \pi r$, we require that both $1 / \chi \rightarrow 0$ and $r \rightarrow 0$ in order for $\lambda n \ll 1$. In other words, that condition can be realized with a relatively short chain of extremely tightly spaced platelets consisting of a material with very large intrinsic magnetic susceptibility $\chi$. Equation (B11) now simplifies to

$$
\lim _{\lambda \rightarrow 0} m(x)=\frac{1}{N_{p}}\left[\left(\frac{n}{2}\right)^{2}-x^{2}\right]+\frac{n}{N_{p}},
$$

where $n / N_{p}=m_{E}$ is the magnetization at either end of the chain in the limit $\lambda \rightarrow 0$. The magnetization $m_{0}=m(x=0)$ of the innermost platelet

$$
\lim _{\lambda \rightarrow 0} m_{0}=\left(1+\frac{n}{4}\right) \frac{n}{N_{p}}=\left(1+\frac{n}{4}\right) m_{E}
$$

is $(1+n / 4)$ times larger than $m_{E}$, which agrees well with the ratio of $(1+n / 2) / 2$ found for the discrete chain. The expression for the average magnetic moment [Eq. (B12)] becomes

$$
\lim _{\lambda \rightarrow 0}\langle m\rangle=\frac{5+6 n+n^{2}}{6 N_{p}},
$$

which again compares well with the value of $(2+3 n$ $\left.+n^{2}\right) /\left(6 N_{p}\right)$ found for the discrete chain. Thus, the total energy

$$
\lim _{\lambda \rightarrow 0} E \cong-\frac{1}{2 N_{p}}(1+n / 6) n^{2} H^{2} p L^{3}
$$

increases at least as fast as $n^{2}$ in contrast to an ensemble of individual particles for which the energy is simply proportional to their number $n$. The same is true for the torque. The reason for such nonlinear response is explained in our preliminary considerations above: a chain of closely spaced platelets can be considered as a single body with large aspect ratio whose demagnetizing factor is much smaller than that of an individual platelet.

Naturally, solution Eq. (20) can be obtained directly from Eq. (B3) when setting $N_{r}^{\prime}=0$. This observation clarifies the physical sense of the condition $\lambda \rightarrow 0$, namely that the magnetostatic self energy of the two surfaces at either end of the chain, which carry uncompensataed poles, dominates over the energy of stray field in the gaps between the platelets.

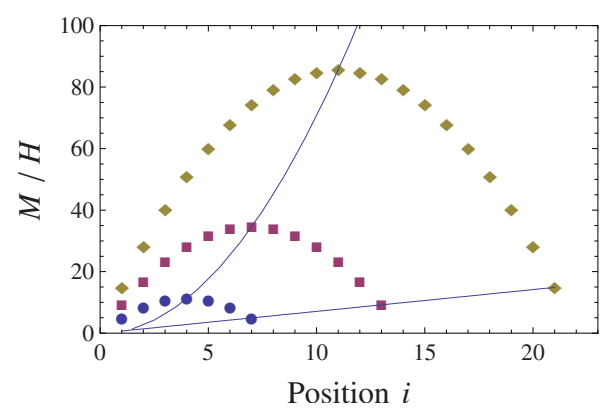

FIG. 5. (Color online) Magnetization distribution $m_{i}=M_{i} / H$ along the position $i$ of a platelet in a chain of $n$ platelets for $1 / \chi$ $=0$ in the limit $r_{i} \rightarrow 0(f \rightarrow 1)$, according to Eq. (16). The curves from the top to the bottom correspond to $n=21$ (diamonds), 13 (squares), 7 (circles) for $p=0.1$. The solid line connecting the central points of each graph increases as $n(1 / 2+n / 4)$, while the one connecting the end points is linear in $n$.

\section{$\lambda n \gg 1$}

For $\lambda n \gg 1$, the magnetization $m(x)$ reduces to $1 / N_{r}^{\prime}$, except at the chain ends, where the magnetization drops to

$$
\lim _{\lambda n \rightarrow \infty} m_{E}=\frac{1}{N_{r}^{\prime}} \frac{1}{1+1 / \lambda}<\frac{1}{N_{r}^{\prime}} .
$$

This behavior has a simple physical meaning: the intensity of the magnetization of each platelet in the inner part of the chain is limited by the magnetostatic self energy $N_{r}^{\prime} m^{2} / 2$ (per platelet) concentrated in the gaps. As the Zeeman energy density of a platelet is $-m$, the minimization of the total energy $-m+N_{r}^{\prime} r m^{2} / 2$ gives exactly $m=1 / N_{r}^{\prime}$.

For $\lambda n \gg 1$, Eq. (B9) transforms to

$$
\lim _{\lambda n \rightarrow \infty} E \cong \frac{1}{2 N_{r}^{\prime}}\left[\frac{N_{p}}{N_{r}^{\prime}(1+1 / \lambda)}-n\right] H^{2} p L^{3} \equiv E_{0}-E_{1} n .
$$

The second term, which is proportional to the number $n$ of platelets, contains the demagnetizing energies of the gaps whereas the first term reflects the demagnetizing energies of the outermost surfaces, which have no counterparts.

\section{RESULTS}

Our mathematical analysis confirms the conclusions drawn from our preliminary considerations, namely that a chain of platelets has the potential to act as a magnetic field amplifier. However, in order to achieve the maximum amplification of the external magnetic field, two conditions have to met. First, the platelets have to be made of magnetic material with high intrinsic susceptibility $\chi$. Second, the gap between the platelets has to be very narrow. When both conditions are met, Eqs. (20)-(23) apply and the magnetization in the central part of the chain is approximately $n(n+2) / 4$ times the external magnetic field, where $n$ is the number of platelets in a chain (see parabolic curve in Fig. 5). Even the magnetization of a platelet at the chain ends is $n$ times larger than the magnetization of an isolated platelet (straight line in Fig. 5).

However, the amplification of the external field decreases drastically as soon as the gaps between the platelets widen, 


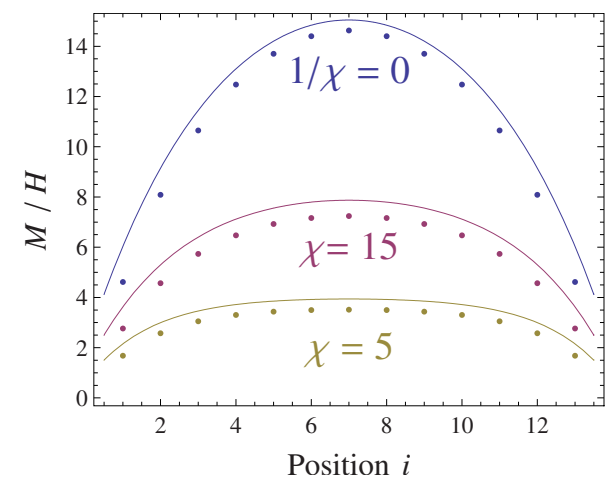

FIG. 6. (Color online) a) Magnetization distribution $m_{i}=M_{i} / H$ along the position $i$ of a platelet in a chain $(n=13, p=0.1)$. As opposed to Fig. 5, the gap size $r$ is finite now (4 nm), i.e., $r$ $=0.004$, or $f=0.97$. The dotted curves are computed according to Eq. (16) (discrete chain), the solid curves represent the continuous magnetization distribution in the chain according to Eq. (B11). A finite intrinsic susceptibility (lower graphs, $\chi=15$ c.g.s and $\chi=5$ c.g.s, respectively) in combination with a finite gap size diminishes the amplification even further.

as exemplified in Fig. 6 for a gap size of $4 \mathrm{~nm}$. The amplification factor diminishes further when both the gap size and the intrinsic susceptibility are finite (lower graphs in Fig. 6). Accordingly, we observe a similar drop in chain energy (Fig. 7). The same applies to the torque. The outcome of these calculations is that in order to achieve a large amplification of the applied field, the gap must be very small, that is, $r$ $\leq 0.005$, or $r L \leq 5 \mathrm{~nm}$, (see Fig. 6). Then, the field in the gap approaches the limit value of $H_{i}=4 \pi M_{i}$. Of course, it is desirable to have platelets with a large intrinsic susceptibility $\chi$. Since $\chi$ is predetermined by the material properties of the

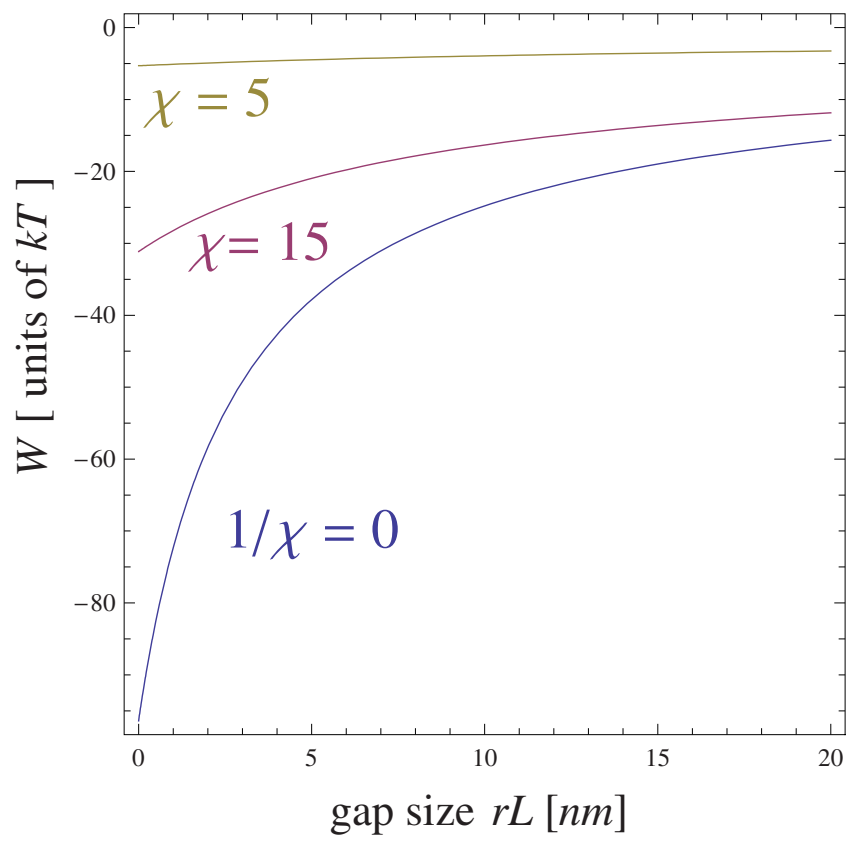

FIG. 7. (Color online) Energy (maximum torque) in units of the thermal energy at $H=0.5 \mathrm{Oe}(40 \mathrm{~A} / \mathrm{m})$ for a chain of $n=13$ platelets as a function of gap width $r$ for various values of the intrinsic susceptibility (in c.g.s.), calculated according to Eq. (16).
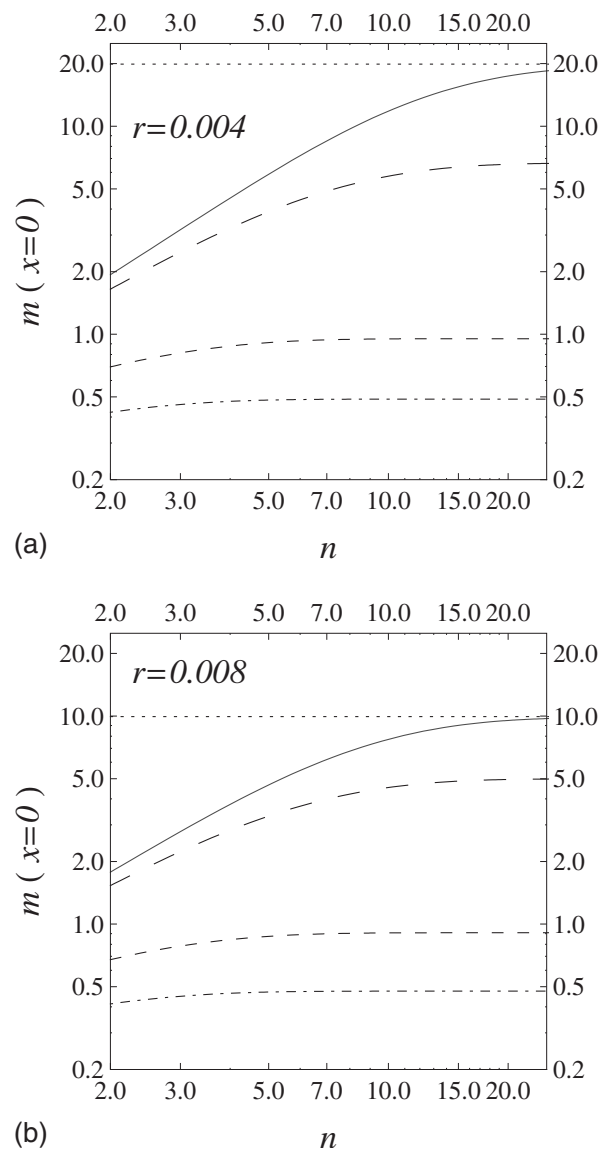

FIG. 8. Amplification factor $\alpha=M / H$ for the central platelets in a chain of $n$ platelets [Eq. (B11)], in function of $n$ for gap width $r L$ of $8 \mathrm{~nm}$ (top) and $4 \mathrm{~nm}$ (bottom), respectively, for various values of $\chi$. From top to bottom: $1 /(4 \pi r)$ (dotted); $1 / \chi=0$ (solid); $\chi=10$ (long dashes); $\chi=1$ (short dashes); $\chi=0.3$ (dot dashed).

platelets, the energy or the torque at given values of $\chi$ and $r L$ can only be increased by increasing the number of platelets in a chain. Doubling the chain length then at least doubles the chain energy.

\section{Magnetization in the center of a chain}

Importantly, $n$ needs to be large enough at a given $r$ and $\chi$ to develop the full amplification potential in the center of the chain. As a rule of thumb, the number $n$ of platelets should exceed the value of the intrinsic magnetic susceptibility $\chi$ in order to reach the asymptotic value of $M(H)$. The maximum magnetization value in a chain occurs in the center of the chain [Eq. (B11)] and is plotted in Fig. 8 in function of $n$ for several values of $\chi$. The asymptote of each curve defines the maximum amplification factor $\alpha_{\infty}$ for a set $n$ and is given by

$$
\alpha_{\infty}=\lim _{n \rightarrow \infty} \frac{M(x=0)}{H}=\left\{\begin{array}{cc}
1 /(4 \pi r), & \chi \gg 1 \\
1 / N_{r}^{\prime}, & \chi>1 . \\
\chi & \chi \leq 1
\end{array}\right.
$$

It is clear from Eq. (25) that amplification is possible only if $\chi>1$. For $\chi \leq 1$, there is no amplification. The amplification factors summarized in Eq. (25) determine the maximum pos- 
sible magnetic attraction force between the two innermost platelets of a chain according to Eq. (13):

$$
F_{\mathrm{ms}} \approx 2 \pi M^{2} p L^{2} \approx 2 \pi \alpha_{\infty}^{2} H^{2} 10^{-2} \mathrm{pN} / \mathrm{Oe}^{2}
$$

Note that for $\chi \leq 1\left(\alpha_{\infty} \leq 1\right)$, the expression for the attraction force between the innermost platelets of a chain, $F_{\mathrm{ms}}$ $\approx 2 \pi \chi^{2} H^{2} p L^{2}$, is similar to the one derived in [15] for the attraction force between two superparamagnetic clusters, $F^{\mathrm{SP}} \sim \chi^{2} H^{2} R^{2}$, where $R$ is the radius of a cluster. For $F_{\mathrm{ms}}$ to reach at least $1 \mathrm{pN}$ at 0.5 Oe (i.e., $\alpha_{\infty} \geq 8$ ), the intrinsic magnetic susceptibility $\chi$ should be at least 15 at $r=4 \mathrm{~nm}$ $(n=15)$. To compensate for an increase in gap size from 4 $\mathrm{nm}$ to $8 \mathrm{~nm}, \chi \geq 40$ and $n \geq 30$ are needed. Because of the limit values of $\alpha$ [Eq. (25)], a smaller $\chi$ cannot be compensated by a much larger number of platelets. It is clear that there is a material-specific limit to $\chi$ and, thus, the only way to reach high amplification factors is to close the gap between adjacent platelets.

\section{DISCUSSION}

The torques and central forces related to the magnetization behavior of a chain of magnetically soft platelets appear suitable to realize a magnetoreception mechanism, provided that the intrinsic magnetic susceptibility is large, say $\chi>10$. That mechanism would be similar to any mechanism based on superparamagnetic particles in two ways. First, the axial orientation of the external field can be detected but not its polarity. This is due to the fact that the polar external field vector is converted into an axial force (deformation). Second, deformation scales with $H^{2}$. However, the distinct advantage of magnetically soft platelets is the drastic amplification of the external field in the narrow gaps due to strong attractive interactions developing between adjacent platelets. An amplification of that magnitude is not possible with interacting spherical clusters of superparamagnetic particles, which have $\chi \leq 1$.

To re-emphasize that point, we recall from our preliminary considerations that the magnetization of an isolated platelet in an external magnetic field $H$ has a limit value of $M=H / N_{p}$. This corresponds to an energy of $H^{2} p L^{3} /\left(2 N_{p}\right)$, which for $L=1 \mu \mathrm{m}, p=0.1$, and $H=0.5$ Oe $(40 \mathrm{~A} / \mathrm{m})$ amounts to $0.6 k_{B} T$. On the other hand, the magnetization of a platelet included in a chain has a limit value of $M$ $=H /\left(r N_{r}\right)$, which for $r=0.01 \ldots 0.001$ exceeds the limit value of $M$ for an isolated platelet by $1 \ldots 2$ orders of value. Correspondingly, the total energy of each platelet in a chain $H^{2} p L^{3} /\left(2 r N_{r}\right)$ exceeds the thermal energy $k_{B} T$ considerably. To allow for this degree of amplification, the gap must be quite narrow, say $r \leq 0.005$, in which case the stray field in the gaps is already close to the limit value of $4 \pi M$ (see Fig. 7 and Table II). Thus, for platelets of size $L=1 \mu \mathrm{m}$, the gap should not greatly exceed $5 \mathrm{~nm}$, which appears to be a reasonable value, as judged from the electron micrographs in [7].

\section{A. Magnetic-field transduction}

In order to convert the maximum external-field amplification produced by the inner platelets in a chain into a physi-

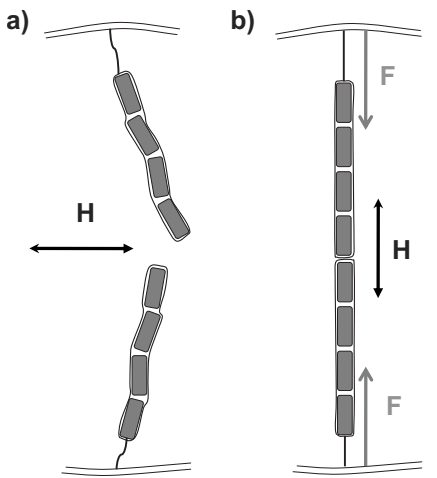

FIG. 9. Relay model. One end of each half chain is attached through filaments to the receptor membrane of the nerve dendrite, the other end is free. a) the external magnetic field is perpendicular to the chain axis, the half chains are not connected. b) the external magnetic field now is parallel to the chain axis. The half chains reconnect across their free ends and form a full chain. The magnetic attraction force emerging this way makes a firm connection, and produces tension in the filaments that connect the chain to the membrane. The membrane pull is proportional to $H^{2}$.

ological stimulus, we propose the "relay" model sketched in Fig. 9. Consider two physically distinct chains of platelets, where each chain is enclosed in a membrane sheath and connected by cytoskeletal filaments to the nerve membrane. If the external field $\mathbf{H}$ is perpendicular to the chain axis, the two chains remain unconnected (open circuit). If, however, the external field $\mathbf{H}$ is parallel to the chain axis, the free endings of the two chains connect (short circuit) because of the attraction force produced by the induced magnetization. The attraction force will be directly transmitted to the membrane of the dendrite, where it can trigger an electrical potential. The attraction force depends quadratically on the external magnetic field strength. The time constant for the connection/disconnection depends on the viscosity of the medium surrounding the chains.

A variant of this magnetic short or relay model is the bonding model (Fig. 10), which takes advantage of the platelets' ability to self-connect into tightly bond linear chains when the magnetic field is parallel to the chain axis. Let us assume that the platelets are free to move along the long axis of the subcellular compartment in which they are enclosed. Under the action of an external magnetic field, the platelets may form chains of different lengths extending in the field direction. We now refer to a chain as a linear arrangement of tightly spaced (bonded) platelets. Due to geometrical constraints (a nerve terminal can be considered a long hollow cylinder), a significant chain growth can happen only along the long axis of the terminal. Now we assume that a platelet at one end of the configuration is connected by a filament to an ion channels in the membrane, while the platelets at the other end is either connected to an ion channel as well or simply anchored to a non-mechanically sensitive element. If all platelets are aligned and the gaps between each pair of adjacent platelets minimized, a magnetic short occurs and the magnetic attraction force can be transmitted to the ion channel(s) through the end platelet(s). As shown in Appendix A, the probability that all platelets of a chain form tight links 


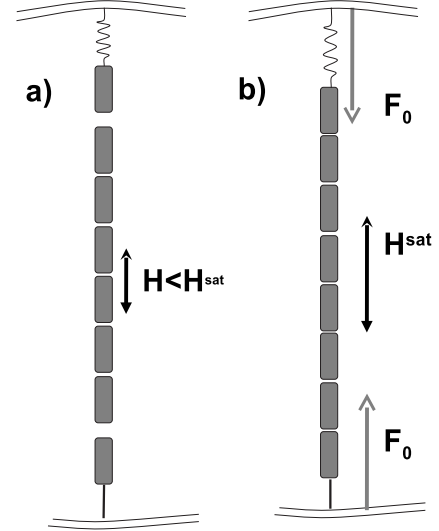

FIG. 10. Bonding model. The stronger the external magnetic field along the chain axis, the higher the probability that all platelets organize into a single chain with tight bonds between adjacent platelets. a) external field smaller than field required for full connection; the end platelets are not connected yet. The transmembrane ion channel is in its rest position. b) at the field strength $H^{\text {sat }}$, the chain is in its fully connected state, which is shorter than the partially connected state. The shortening implies tension and can be used to fully open a stretch-sensitive transmembrane ion channel or, as shown schematically here, a force-gated ion channel with a gating spring that extends upon pulling. See Fig. 11 for the opening probability of the ion channel in function of the external field strength.

with their neighbors at field strength $H$ is given by

$$
P(H)=\frac{\exp (-\gamma)}{\{1+\exp [-\beta(H)]\}^{n}+\exp (-\gamma)-1},
$$

where $\beta=E_{0}(H) / k_{B} T$ and $\gamma=E_{\text {gate }} / k_{B} T$ with $E_{\text {gate }}$ being the energy required to open all ion channels (gates) connected to the chain of platelets. By reciprocity, $P(H)$ according to Eq. (27) gives also the opening probability of ion channels, since full connection can happen only if the ion channels open. The energy required to open an individual ion channel is typically a few $k_{B} T$ (e.g., [37]). Generally, $P(H)$ rises sharply in a narrow field range of less than 0.1 Oe width (Fig. 11). The transition range increases for a larger gap size. Within the transition range, the state of the chain varies constantly being fully and partly connected. A persistent fully connected chain exists once $P(H)$ saturates [e.g., 0.2 Oe for the solid
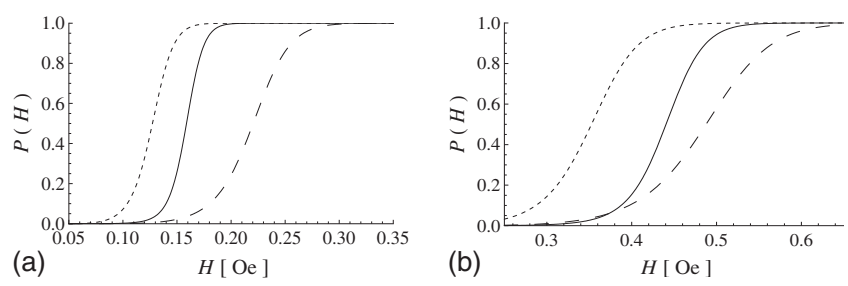

FIG. 11. Probability $P(H)$ for ion channels to open in the bonding model shown in Fig. 10 as a function of the magnetic field strength $H$ according to Eq. (27) for $n+1=16$ platelets ( $L$ $=1 \mu \mathrm{m}, p=0.1 L)$. a) for $\beta=E_{0}(H) / k_{B} T$, b) for $\beta=E_{1}^{\prime}(H) / k_{B} T$. $E_{\text {gate }}=7 k_{B} T$ and $r L=2 \mathrm{~nm}$ (solid); $E_{\text {gate }}=3.5 k_{B} T$ and $r L=4 \mathrm{~nm}$ (long dashes); $E_{\text {gate }}=3.5 k_{B} T$ and $r L=2 \mathrm{~nm}$ (short dashes). curve in Fig. 11(a)]. Note that the magnetostatic coupling between end platelet and its nearest neighbor may be smaller than the restoring force by which the end platelet is pulled toward the ion channel, away from its nearest neighbor in the chain. In that case, we have a weak link and $\beta=E_{0}(H) / k_{B} T$ in Eq. (27) underestimates the field strength required for the fully connected state. Therefore, to be on the safe side, we need to find the field strength at which the end platelet is bound to its nearest neighbor. This is shown in the curves in Fig. 11(b), for which $\beta=E_{1}^{\prime}(H) / k_{B} T$, where $E_{1}^{\prime}(H)$ is $E_{1}$ from Eq. (24) plus the energy due to an isolated platelet, $p L^{3} H^{2} /\left(2 N_{p}\right)$

\section{B. Comparison with the conceptual model by [22]}

From our analysis we can now turn to assessing the magnetic flux distribution proposed in [22] (Fig. 1), where a chain of $n$ soft platelets is interrupted at position $i$ by a cluster of superparamagnetic particles. The interruption means that the gap size is very large and the two platelets $i$ and $i$ +1 either side of the large gap do practically not interact. In other words, they behave just as end platelets and their maximum possible magnetization will be $m_{E}=(n-i) / N_{p}$ and $m_{E}$ $=(n-i-1) / N_{p}$ in the optimal case that the platelets in each subchain of length $n-i$ and $n-i-1$ are extremely tightly spaced and moreover are made of a very soft magnetic material [see Eq. (20)]. The presence of a superparamagnetic cluster with a much smaller intrinsic susceptibility $\chi \leq 1$ will not help to amplify the magnetic field in the large gap. The same considerations apply to the case where the superparamagnetic cluster is at the end of a chain. Thus, the model proposed in [22] and depicted in Fig. 1 is viable only if the platelets consist of a ferro(i)magnetic material with a large spontaneous magnetization (such as maghemite or magnetite) that is capable of producing a strong stray field of its own.

\section{Biological context}

Before putting our results in a biological context, we emphasize that the following considerations apply to the optimistic case where the intrinsic susceptibility is large enough to produce noticeable effects. The case of vanishing susceptibility only allows the trivial conclusion that the platelets are not magnetically active in the putative magnetoreceptor structures.

\section{Measurement of magnetic intensity and functional window}

The magnetic forces resulting from the interactions between the platelets depend quadratically on the intensity of the external magnetic field. Therefore, a potentially relevant application of these mechanisms is determination of magnetic-field intensity. Although it is not clear which one of the geomagnetic elements act as factor in the navigational map [3], geomagnetic intensity and its spatial variations are deemed suitable as map co-factors [38]. As shown in Fig. 11, the system can respond to a change in intensity most sensitively where the open probability of ion channels is $50 \%$. That point defines a suitable operation point for determining 
variations in field intensity. The transition from a few percent open probability to nearly saturation occurs over a small field range. The bird would have to adjust the set point when it enters a geographic region with different intensity baseline. The set-point principle is generally in accord with a narrow intensity window within which the magnetic compass sense works. It was shown experimentally that the intensity window can be adapted fast to a field strength outside the normally encountered range [39].

\section{Measurement of magnetic-field direction}

Due to the pronounced shape anisotropy imparted by the chain structure, the platelets could be used to determine the axial orientation of the field lines, but not their polarity. It has been inferred from behavioral experiments on birds tested under abnormal light conditions that the beak mediates orientation responses that depend on the field polarity (see [40] for a thorough discussion). To realize a polarity compass, magnetic remanence is necessary, which acts as a polarity bias $[41,42]$. Thus far, however, there is no direct (microscopic) evidence of remanence bearing structures in the candidate magnetoreceptors in the beak of pigeons, although remanence stable at room temperature has been detected in bulk magnetic measurements of pigeon beaks [43].

\section{Effect of a magnetic pulse}

Experimental support for the involvement of magnetite in the magnetic sense in birds, bats, and sea turtles has been provided by orientation experiments on test animals subjected to a brief but strong magnetic pulse. The temporary change in the direction of orientation is consistent with ferro(i)magnetic material [44-48]. It is obvious that a magnetic pulse applied parallel to a chain of platelets will magnetically saturate the chain. The interaction field will be two orders of magnitude higher than in the Earth's field. If the direction of the pulse field does not lie along the chain axis, magnetic moments will be induced perpendicular to the long axis and produce strong repulsive forces. As a consequence, a number of chains will be just destroyed.

\section{Possible effects of RF fields}

The radio frequency $(\mathrm{RF})$ fields used in the behavioral experiments mentioned to specifically knock out an axial compass mechanism based on radical pair processes $[49,50]$ had frequencies $f$ in the $1-10 \mathrm{MHz}$ range and amplitudes $\mu_{0} H_{\mathrm{RF}}$ in the range $85-485 \mathrm{nT}(0.85-4.85 \mathrm{mOe})$. The power $P$ dissipated in a chain of $n$ platelets is

$$
P=\frac{n f \operatorname{Im}[\chi] H^{2} p L^{3}}{4 \pi},
$$

where $\operatorname{Im}[\chi]$ is the dissipative part of the (complex) magnetic susceptibility. Taking $n=10, f=10^{6} / \mathrm{s}, H_{\mathrm{RF}}=1 \mathrm{mOe}$, and $\operatorname{Im}[\chi]=0.01$, we obtain $P=0.2 k_{B} T / \mathrm{s}$. A typical experiment lasts $1 \mathrm{~h}$, that is, the birds are exposed to the RF radiation for $1 \mathrm{~h}$, during which time the platelets can dissipate enough energy to heat their environment. The surplus heat, if not transported away by the blood stream, can inactivate fila- ments or other structures involved in the mechanic transduction system. However, we expect energy dissipation to occur independent of the orientation of the RF-field vector $\mathbf{H}_{\mathrm{RF}}$ with respect to the static field $\mathbf{H}$. This expectation is in contrast to the experimental results by [49], who showed that $\mathbf{H}_{\mathrm{RF}}$ must have a component perpendicular to $\mathbf{H}$ in order to impair the magnetic compass sense of birds. We would therefore have to invoke a pronounced anisotropy in $\operatorname{Im}[\chi]$ to explain the results from the RF experiments in terms of the platelets.

\section{CONCLUSIONS}

There is good biological evidence that the iron-bearing terminals in the upper beak are magnetoreceptors. Unfortunately, no direct information about the magnetic properties of the iron-containing structures is available thus far. While technical nanoparticle systems such as ferrofluids may present adequate model systems to describe the properties of the superamagnetic nanocrystals in the clusters and to constrain their magnetic susceptibility, the magnetic nature of the conspicuous bands of platelets is totally open. While the histological context suggests an active involvement of the platelets in magnetoreception, diffraction images have revealed the absence of a crystalline structure in the platelets. Without a crystalline structure, the magnetic compound in the platelets has no magnetocrystalline anisotropy energy and thus is most likely to behave magnetically soft. The single-most important magnetic parameter for such a material is the intrinsic magnetic susceptibility, $\chi$. We have derived analytical expressions for the equilibrium magnetization in a chain of platelets for arbitrary values of $\chi$ in function of chain length and gap width, for platelet dimensions similar to the ones reported for in the avian dendrites. An adaptation to different geometries can be done easily by adapting the demagnetizing factors. From our analysis we conclude that $\chi$ has to be larger than unity (in c.g.s.) in order to obtain an effective magnetoreception mechanism. Of course, the larger $\chi$, the more sensitive the mechanism can respond to small changes in magnetic field strength, in which case a magnetoreceptor mechanism can exploit attraction forces between adjacent platelets in a linear chain.

\section{ACKNOWLEDGMENTS}

Our work was supported by the Human Frontier Science Program (Grant No. RGP28/2007) and the Deutsche Forschungsgemeinschaft (Grant No. Wi1828/4-1).

\section{APPENDIX A: THERMODYNAMICS OF A CHAIN}

To calculate the free energy of Eq. (16),

$$
e=-\sum_{i=1}^{n} m_{i}+2 F_{p} \times\left[\left(1+\frac{1}{N_{p} \chi}\right) \sum_{i=1}^{n} m_{i}^{2}-\sum_{i=1}^{n-1} f\left(p, r_{i}\right) m_{i} m_{i+1}\right] \text {, }
$$

we apply the transformation $\mathbf{x}=\mathbf{A} \cdot \mathbf{m}$, which reduced the quadratic form to principal axes, 


$$
e=-\sum_{i=1}^{n}\left[\sum_{k=1}^{3} a_{k i} x_{i}+2 F_{p} \lambda_{i}(p, \mathbf{r}) x_{i}^{2}\right],
$$

where $\lambda_{i}(p, \mathbf{r})$ are the eigenvalues of the symmetric tridiagonal matrix $\mathbf{W}$,

$$
\mathbf{W}=\left(\begin{array}{ccccc}
2 s & -f\left(p, r_{1}\right) & 0 & 0 & \ldots \\
-f\left(p, r_{1}\right) & 2 s & -f\left(p, r_{2}\right) & 0 & \ldots \\
0 & -f\left(p, r_{2}\right) & 2 s & -f\left(p, r_{3}\right) & \ldots \\
\vdots & \vdots & \vdots & \vdots & \ddots \\
0 & 0 & 0 & 0 & \ldots
\end{array}\right)
$$

with

$$
s=\frac{(1+N \chi)}{N \chi} .
$$

The transformation matrix $\mathbf{A}=\left\{a_{i k}(p, \mathbf{r})\right\}$ may be found by compounding the orthogonal normalized eigenvectors $\mathbf{a}_{i}$ of $\mathbf{W}$ into a matrix whose columns are $\mathbf{a}_{i}$. Then Eq. (A2) can be rearranged as

$$
e=\sum_{i=1}^{n}\left[F_{p} \lambda_{i}\left(x_{i}-\frac{b_{i}}{2 F_{p} \lambda_{i}}\right)^{2}-\frac{b_{i}^{2}}{2 F_{p} \lambda_{i}}\right],
$$

where the coefficients

$$
b_{i}=\sum_{k} a_{k i}
$$

The partition function is

$$
\begin{aligned}
Z= & \prod_{i}^{n}\left\{\exp \left(\alpha \frac{b_{i}^{2}}{4 F_{p} \lambda_{i}}\right)\right. \\
& \left.\times \int_{-\infty}^{\infty} \exp \left[-\alpha F_{p} \lambda_{i}\left(x_{i}-\frac{b_{i}}{2 F_{p} \lambda_{i}}\right)^{2}\right] d x_{i}\right\} \\
= & \prod_{i}^{n} \exp \left(\alpha \frac{b_{i}^{2}}{4 F_{p} \lambda_{i}}\right) \sqrt{\frac{\pi}{\alpha F_{p} \lambda_{i}}},
\end{aligned}
$$

where the parameter

$$
\alpha=\frac{p L^{3} H^{2}}{k_{B} T},
$$

characterizes the relative strength of the external field energy in relation to the thermal energy. The free energy $F=$ $-k_{B} T \ln Z$ is finally obtained as

$$
F(H, p, \mathbf{r}, T)=U-\sum_{i=1}^{n} L^{3} p H^{2} \frac{b_{i}^{2}}{4 F_{p} \lambda_{i}},
$$

where

$$
U=\frac{k_{B} T}{2}\left[\ln \frac{\pi}{\alpha F_{p}}-\ln (\operatorname{det} \mathbf{W})\right]
$$

and det $\mathbf{W}=\Pi_{i} \lambda_{i}$. Note that $U$ represents the internal energy of the chain due to the existence of internal degrees of freedom, which here are related to the possibility that the mag- netization of each individual platelet can formally assume any value as indicated by the integral limits of Eq. (A4). From Eq. (A4), it immediately follows that the thermodynamic average values for the (transformed) magnetization,

$$
\left\langle x_{i}\right\rangle=\frac{b_{i}}{2 F_{p} \lambda_{i}},
$$

exactly match the mechanical equilibrium values

$$
x_{i}=\frac{b_{i}}{2 F_{p} \lambda_{i}},
$$

resulting from the energy function Eq. (A3).

\section{APPENDIX B: CONTINUOUS MAGNETIZATION DISTRIBUTION}

To derive an analytical solution for the magnetization distribution in a chain of soft platelets, we need to transform the discrete system into a continuous one. First, we rearrange Eq. (16) into

$$
\begin{aligned}
e= & -\sum_{i=1}^{n} m_{i}+\frac{1}{4} N_{p}\left\{m_{1}^{2}+m_{n}^{2}+\sum_{i=1}^{n-1}\left(m_{i+1}-m_{i}\right)^{2}\right. \\
& \left.+2 \sum_{i=1}^{n-1} m_{i} m_{i+1}\left[1-f\left(p, r_{i}\right)\right]\right\}+\frac{1}{2 \chi} \sum_{i=1}^{n} m_{i}^{2},
\end{aligned}
$$

With the demagnetizing factor of a gap introduced in Eqs. (11) and (B1) can be recast into

$$
\begin{aligned}
e= & -\sum_{i=1}^{n} m_{i}+\frac{1}{4} N_{p}\left\{2 m_{E}^{2}+\sum_{i=1}^{n-1}\left(m_{i+1}-m_{i}\right)^{2}\right\}+\sum_{i=1}^{n-1} m_{i}\left[m_{i}\right. \\
& \left.+\left(m_{i+1}-m_{i}\right)\right] \frac{r_{i} N_{r}}{2}+\frac{1}{2 \chi} \sum_{i=1}^{n} m_{i}^{2},
\end{aligned}
$$

where we set $m_{E} \equiv m_{1}=m_{n}$, since the magnetization is reflection symmetric about the center of the chain. For the same reason, the term $\sum_{i=1}^{n-1} m_{i}\left(m_{i+1}-m_{i}\right)$ does not contribute to the total energy. Replacing now the summation by an integral, we obtain the total energy in the continuous representation as

$$
e=2 \int_{0}^{n / 2}\left[\frac{N_{p}}{4}\left(\frac{d m}{d x}\right)^{2}+\frac{N_{r}^{\prime}(x)}{2} m^{2}-m\right] d x+\frac{N_{p}}{2} m_{E}^{2},
$$

where the integration limits $x=0$ and $x=n / 2$ correspond to the center and the end, respectively, of the chain. The variable $N_{r}^{\prime}$ represents the effective demagnetizing factor of a gap at position $x$, defined by

$$
N_{r}^{\prime}=\frac{1+\chi r N_{r}}{\chi} .
$$

In the limit of $1 / \chi \rightarrow 0, N_{r}^{\prime} \rightarrow r N_{r}$, which appears to be inconsistent with the definition of the demagnetizing factor $N_{r}$ of a discrete gap given in Eq. (11). In the continuous representation, however, $r(x)$ should be considered as the proportion of 
the gap length to the particle length at position $x$, in other words, $r(x)$ is the weight of $N_{r}$. Variation of Eq. (B3) leads to the following Euler equation

$$
\frac{N_{p}}{2 N_{r}^{\prime}(x)} \frac{d^{2} m}{d x^{2}}-m+\frac{1}{N_{r}^{\prime}(x)}=0 .
$$

The boundary conditions are,

$$
\left.m\right|_{x=n / 2}=m_{E} \quad \text { and }\left.\quad \frac{d m}{d x}\right|_{x=0}=0,
$$

For the sake of simplicity, we let $r(x)=r$ and obtain the solution to Eq. (B5) as

$$
m(x)=\frac{1}{N_{r}^{\prime}}-\left[\left(\frac{1}{N_{r}^{\prime}}-m_{E}\right) \frac{\cosh \lambda x}{\cosh \lambda n / 2}\right] .
$$

where $\lambda$ is the characteristic nondimensional length scale of the problem,

$$
\lambda=\sqrt{\frac{2 N_{r}^{\prime}}{N_{p}}} .
$$

Putting Eq. (B7) in Eq. (B3), we obtain the total energy as

$$
e=\frac{N_{p}}{2} m_{E}^{2}+\frac{1}{N_{r}^{\prime}}\left[\frac{\left(1-N_{r}^{\prime} m_{E}\right)^{2} \tanh (\lambda n / 2)}{\lambda}-\frac{n}{2}\right] .
$$

The magnetization $m_{E}$ at the edge of the chain (at $x$ $= \pm n / 2)$ can now be determined by requiring that $e\left(m_{E}\right)$ is minimum, from which we obtain

$$
m_{E}=\frac{1}{N_{r}^{\prime}}\left(\frac{\lambda}{\lambda+\operatorname{coth} \lambda n / 2}\right),
$$

so that the final solution $m(x)$ is given by

$$
m(x)=\frac{1}{N_{r}^{\prime}}\left(1-\frac{\cosh \lambda x}{\cosh \lambda n / 2+\lambda \sinh \lambda n / 2}\right) .
$$

To obtain the average magnetization of the chain, $\langle m\rangle$, which determines both total magnetic energy and torque, we integrate Eq. (B11),

$$
\langle m\rangle=\frac{2}{n} \int_{0}^{n / 2} m(x) d x=\frac{1}{N_{r}^{\prime}}\left(1-\frac{2}{\lambda^{2} n+\lambda n \operatorname{coth} \lambda n / 2}\right) .
$$

\section{APPENDIX C: SELF-ORGANIZATION OF PLATELETS INTO A LINEAR CHAIN}

We consider an ensemble of $n+1$ identical platelets, which are capable of forming one-dimensional chains of various length. Accordingly, there are $n$ possible links between the platelets. Let $m$ the number of broken links, so that there are $m+1$ segments (subchains) in the entire ensemble. From Eq. (24), the total magnetic energy of a connected chain of length $n$ is $E_{0}-E_{1} n$, where $E_{0}=N_{p} H^{2} p L^{3} /\left(2 N_{r}^{\prime 2}\right)$ and $E_{1}=H^{2} p L^{3} /\left(2 N_{r}^{\prime}\right)$. Hence, the total energy of the ensemble containing $n+1$ platelets with broken links (i.e., $m$ +1 segments) is given by

$$
E_{m}=-n E_{1}+m E_{0}-E_{1}+E_{0},
$$

which is independent of the distribution of breaks over the chain. Because the number of possible states is

$$
C_{m}^{n}=\frac{n !}{m !(n-m) !},
$$

the total energy of all states having $m$ broken links is

$$
E_{m}=\frac{n !}{m !(n-m) !} m E_{0} .
$$

Omitting the insignificant constant term in $E_{m}$, the partition function of such a system is

$$
Z=\sum_{m=0}^{n} \frac{n !}{m !(n-m) !} \exp (-\beta m)=[1+\exp (-\beta)]^{n} .
$$

where $\beta=E_{0} / k_{B} T$. To assess the probability of opening mechanosensitive ion channels, we take into account the additional energy $E_{\text {gate }}=\gamma k_{B} T$ required to open them. This changes Eq. (C1) to

$$
\begin{aligned}
Z= & \sum_{m=0}^{n} \frac{n !}{m !(n-m) !} \exp (-\beta m)+\exp (-\gamma)=[1+\exp (-\beta)]^{n} \\
& +\exp (-\gamma)-1 .
\end{aligned}
$$

Thus, the probability $P$ that a chain containing $n+1$ is fully connected (no broken links) and the ion channels are open is

$$
P=\frac{\exp (-\gamma)}{[1+\exp (-\beta)]^{n}+\exp (-\gamma)-1} .
$$

[1] V. P. Bingman and K. Chen, Ethol. Ecol. Evol. 17, 295 (2005).

[2] W. Wiltschko and R. Wiltschko, J. Comp. Physiol. [A] 191, 675 (2005).

[3] M. J. Freake, R. Muheim, and J. B. Phillips, Q. Rev. Biol. 81, 327 (2006).

[4] M. M. Walker, C. E. Diebel, C. V. Haugh, P. M. Pankhurst, and J. C. Montgomery, Nature (London) 390, 371 (1997).

[5] C. E. Diebel, R. Proksch, C. R. Green, P. Neilson, and M. M. Walker, Nature (London) 406, 299 (2000).
[6] M. Winklhofer, E. Holtkamp-Rötzler, M. Hanzlik, G. Fleissner, and N. Petersen, Eur. J. Mineral. 13, 659 (2001).

[7] G. Fleissner, E. Holtkamp-Rötzler, M. Hanzlik, M. Winklhofer, G. Fleissner, N. Petersen, and W. Wiltschko, J. Comp. Neurol. 458, 350 (2003).

[8] R. C. Beason and P. Semm, J. Exp. Biol. 199, 1241 (1996).

[9] C. V. Mora, M. Davison, M. J. Wild, and M. M. Walker, Nature (London) 432, 508 (2004).

[10] W. Wiltschko, U. Munro, H. Ford, and R. Wiltschko, Proc. R. 
Soc. B 276, 2227 (2009).

[11] P. Semm and R. C. Beason, Brain Res. Bull. 25, 735 (1990).

[12] H. Cadiou and P. A. McNaughton, J. R. Soc. Interface 7, S193 (2010).

[13] M. Zapka et al., Nature (London) 461, 1274 (2009).

[14] M. Hanzlik, C. Heunemann, E. Holtkamp-Rötzler, M. Winklhofer, N. Petersen, and G. Fleissner, BioMetals 13, 325 (2000).

[15] A. F. Davila, G. Fleissner, M. Winklhofer, and N. Petersen, Phys. Chem. Earth 28, 647 (2003).

[16] V. P. Shcherbakov and M. Winklhofer, Phys. Rev. E 70, 061803 (2004).

[17] A. F. Davila, M. Winklhofer, V. P. Shcherbakov, and N. Petersen, Biophys. J. 89, 56 (2005).

[18] M. Winklhofer, IEEE Trans. Magn. 45, 5259 (2009).

[19] B. Stahl, G. Fleissner, G. Falkenberg, and G. Fleissner, in Proceedings of the 4th Fall Conference on Metalloproteins and Metalloidproteins, edited by A. Kyriakopoulos, B. Michalke, A. Graebert, and D. Behne (Herbert Utz Verlag, München, 2006), pp. 63-68.

[20] G. Fleissner, B. Stahl, P. Thalau, G. Falkenberg, and G. Fleissner, Naturwiss. 94, 631 (2007).

[21] M. Winklhofer and J. L. Kirschvink, arXiv:0805.2249 (unpublished).

[22] G. Fleissner, B. Stahl, P. Thalau, G. Falkenberg, and G. Fleissner, J. Ornithol. 148, S643 (2007).

[23] S. Johnsen and K. J. Lohmann, Phys. Today 61(3), 29 (2008).

[24] I. A. Solov'yov and W. Greiner, Biophys. J. 93, 1493 (2007).

[25] I. A. Solov'yov and W. Greiner, Eur. Phys. J. D 51, 161 (2009).

[26] B. Stahl, G. Fleissner, G. Fleissner, and E. Holub-Krappe, in Micromagnetic Aspects of Magnetoreception of Homing Pigeons Based on Iron Minerals, Vol 882 of AIP Conf. Proc. (AIP, Melville, NY, 2007), pp. 755-757.

[27] I. A. Solov'yov and W. Greiner, Phys. Rev. E 80, 041919 (2009).

[28] P. Rhodes and G. Rowlands, Proc. Leeds Philos. Lit. Soc. 6, 191 (1954).

[29] A. Hubert and R. Schäfer, Magnetic Domains (Springer, Berlin, 1998).

[30] A. J. Newell, W. Williams, and D. J. Dunlop, J. Geophys. Res.
98, 9551 (1993).

[31] M. J. Donahue and D. G. Porter, Interagency Report NISTIR 6376, National Institute of Standards and Technology, Gaithersburg, MD, 1999 (unpublished).

[32] W. Scholz, K. Y. Guslienko, V. Novosad, D. Suess, T. Schrefl, R. W. Chantrell, and J. Fidler, J. Magn. Magn. Mater. 266, 155 (2003).

[33] V. P. Shcherbakov and G. P. Markov, Izv. Akad. Nauk. USSR, Phys. Solid Earth 18, 681 (1982).

[34] D. J. Dunlop, Geophys. Res. Lett. 10, 79 (1983).

[35] S. Xu and R. T. Merrill, J. Geophys. Res. 92, 10657 (1987).

[36] V. P. Shcherbakov, M. Winklhofer, M. Hanzlik, and N. Petersen, Eur. Biophys. J. 26, 319 (1997).

[37] J. Howard, Mechanics of Motor Proteins and the Cytoskeleton (Sinauer Associates, Inc., Sunderland, 2001).

[38] K. J. Lohmann, N. F. Putman, and C. M. F. Lohmann, Proc. Natl. Acad. Sci. U.S.A. 105, 19096 (2008).

[39] W. Wiltschko, K. Stapput, P. Thalau, and R. Wiltschko, Naturwiss. 93, 300 (2006).

[40] R. Wiltschko, K. Stapput, P. Thalau, and W. Wiltschko, J. R. Soc. Interface 7, S163 (2010).

[41] J. L. Kirschvink, M. Winklhofer, and M. M. Walker, J. R. Soc. Interface 7, S179 (2010).

[42] M. Winklhofer and J. L. Kirschvink, J. R. Soc. Interface 7, S273 (2010).

[43] L. Tian, B. Xiao, W. Lin, S. Zhang, R. Zhu, and Y. Pan, BioMetals 20, 197 (2007).

[44] R. Wiltschko, U. Munro, R. C. Beason, H. Ford, and W. Wiltschko, Experientia 50, 697 (1994).

[45] R. C. Beason, N. Dussourd, and M. E. Deutschlander, J. Exp. Biol. 198, 141 (1995).

[46] W. P. Irwin and K. J. Lohmann, J. Comp. Physiol. [A] 191, 475 (2005).

[47] W. Wiltschko, H. Ford, U. Munro, M. Winklhofer, and R. Wiltschko, J. Comp. Physiol. [A] 193, 515 (2007).

[48] R. A. Holland, J. L. Kirschvink, T. G. Doak, and M. Wikelski, PLoS ONE 3, e1676 (2008).

[49] T. Ritz, P. Thalau, J. Phillips, R. Wiltschko, and W. Wiltschko, Nature (London) 429, 177 (2004).

[50] P. Thalau, T. Ritz, H. Burda, R. E. Wegner, and R. Wiltschko, J. R. Soc., Interface 3, 583 (2006). 